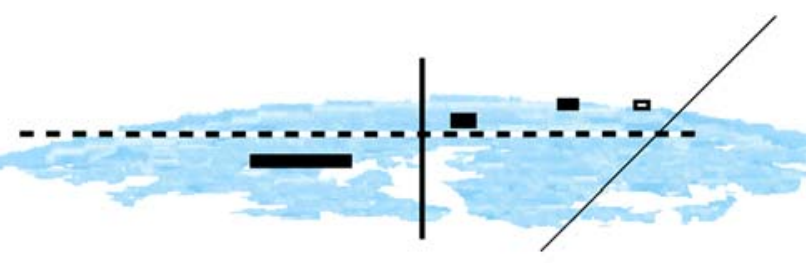

Geoforschungs Zenthum Potsdam STIFTUNG DES OFFENTLICHEN RECHTS

\title{
Scientific Technical Report
}

ISSN 1610-0956 


\title{
Polar motions with a half-Chandler period and less in their temporal variability
}

\author{
Joachim Höpfner \\ GeoForschungsZentrum Potsdam (GFZ), Division Kinematics and Dynamics of the Earth, Telegrafenberg, 14473 \\ Potsdam, Germany, e-mail: ho@gfz-potsdam.de
}

\begin{abstract}
Our study focuses on the observed higher-frequency polar motions that are substantially smaller than the Chandler and annual wobbles. Here, the combined Earth orientation series SPACE99 from 1976 to 2000 with one-day sampling is used as input data, after removing the low-frequency, the Chandler and annual terms. We applied a data processing procedure including four steps, each computing the amplitude spectrum by a Fast Fourier Transform in order to reveal the periodic signals in the residual motions, and then separating their components from the residual time series by band-pass filtering. In particular, the oscillations have the following periods: Semi-Chandler and semi-annual periods and those of order four, three, two, and one and a half months, as well as quasi-biennial and 300-day periods. We show to what extent the observed polar motions are irregularly occurring. A very small polar motion signal with the period of one month is still found in the remaining motions.
\end{abstract}

Key words: Polar motion, periodic components, semi-Chandler wobble, semi-annual wobble, and 4-month, 90-day, 2-month and 1.5-month components, quasi-biennial and 300-day components, magnitude, variability

\section{Introduction}

Irregularities in the Earth's rotation occur in both the direction of the rotation axis relative to a fixed reference frame in space (precession and nutation motions) and to the Earth and in the rate of rotation over many time scales. The axis orientation relative to the Earth and the temporal rotation variations are measured by polar motion (PM) and changes in length-of-day (LOD). The study and monitoring of the motion of the Earth's rotation pole began in 1899 with the establishment of the International Latitude Service (ILS) (Helmert and Albrecht, 1899). Initially, PM solutions were obtained from latitude observations made by the use of visual zenith telescopes (VZTs). Since then, new methods have allowed an improvement in the precision of such measurements by more than a factor of 100. Specially, measurement uncertainties which were of the order of a few tenths of an arcsecond in the past are now ca. 0.1 milliarcseconds (mas).

The time resolution of these measurements has also increased with the measurement precision. Concerning the sampling of PM data from the mid-19th century to the present, some specific examples should be noted. The first ILS time series of polar motion were calculated for different periods from 1900.0 at 0.1 -year intervals, for example, Albrecht and Wanach (1909), Wanach (1916) and Wanach and Mahnkopf (1932). Next, there are PM solutions at 1/12-year intervals, for example Nicolini and Fichera (1970). Yumi and Yokoyama (1980) re-reduced all ILS observations in a consistent way. Also their homogeneous ILS time series computed for the period 1899.9-1979.0 is given at 1/12-year intervals.

From the International Earth Rotation Service (IERS), four combined Earth orientation parameter (EOP) solutions are available, namely EOP(IERS) C01 since 1846 at 0.05-year intervals, EOP(IERS) C02 since 1962 at 5-day intervals, EOP (IERS) C03 since 1993 at 1-day intervals and EOP(IERS) C04 since 1962 at 1-day intervals (for example, see IERS 1999). More information on the ILS and non-ILS time series including their sampling can be found in Höpfner (2000) and references therein.

Based on past latitude observations, only polar motions with relatively large amplitudes and longer periods such as the Chandler and annual wobbles are observable (see e. g. Höpfner 2002). There are polar motions at frequencies higher than the Chandler and annual wobbles, but these have a substantially smaller amplitude. Therefore, their study had to wait for improved data series, in particular these that were based on high-precision and high-resolution space-geodetic 
Publication: Scientific Technical Report

No.: 02/13

Author: J. Höpfner

Table 1. Parameters of the ellipses of the semi-Chandler and semi-annual wobbles obtained from the IERS data for the period 1976.5 to 1988.0

\begin{tabular}{lcccc}
\hline Wobble & $\begin{array}{c}\text { Period } \\
\text { (days) }\end{array}$ & $\begin{array}{c}\text { Semi-major axis } \\
\text { (mas) }\end{array}$ & $\begin{array}{c}\text { Direction of the } \\
\text { semi-major axis }\end{array}$ & $\begin{array}{c}\text { Semi-minor axis } \\
\text { (mas) }\end{array}$ \\
\hline Semi-Chandler & 206.19 & $2.82 \pm 0.66$ & $162.77^{0} \pm 12.76^{0}$ & $0.50 \pm 0.67$ \\
Semi-annual & 182.62 & $5.05 \pm 0.59$ & $32.15^{0} \pm 7.25^{0}$ & $1.10 \pm 0.59$ ) \\
\hline
\end{tabular}

techniques. As a consequence of the superior measurements, other PM oscillations were revealed with periods ranging from a few hours to many years. For example, Kosek et al. (1995) discuss motions with periods ranging between 20 to 150 days and magnitudes up to 2 to 6 mas. In Kołaczek et al. (2000), the sub-seasonal oscillations are studied, in particular those with periods of 120,62 , and 49 days.

Two other oscillations that have received considerable attention are those with a semi-annual and a half-Chandler periods. There are studies relating to these made already twenty years ago using latitude observations, for example Emetz $(1979,1980)$ and Höpfner $(1982,1985)$. Moreover, since the mid-nineties, the objective has been to analyse both oscillations using time series of polar motion. Some results are presented in Höpfner $(1995,1996 a)$ for the analysis interval between 1976.5 to 1988.0 and Kosek and Kołaczek (1997) for the interval between 1962.0 to 1996.0. Some details on these studies should be noted.

Based on 30 time series of latitude observations made at 22 observatories between 1900 to 1969 (Fedorov et al. 1972), Emetz $(1979,1980)$ studied the spectrum of the latitude variations in the frequency range 1.4 to 2.2 cycles per year (cpy). Besides the semi-annual period, oscillations with periods of 0.54, 0.58, 0.60 and 0.64 years were resolved, which are equal to a half the periods found in the Chandler frequency band, in particular 1.10, 1.17, 1.20, and 1.24 years. Concerning the magnitude of the half-Chandler wobble, an amplitude of ca. 0.0037" was calculated (Höpfner 1983) from the $\mathrm{x}$ - and $\mathrm{y}$-coordinates published in Emetz (1979).

Höpfner (1983) analysed the time series of the latitude changes obtained from observations made with a Danjon astrolabe at the Potsdam Geodetic-Astronomical Observatory between 1957.8 to 1978.0, with the temporal variations in the mean latitude and in the Chandler, annual and semi-annual wobbles discussed in Höpfner (1977, 1979). The remaining latitude time series, after removing the mean latitude and Chandler, annual and semi-annual wobbles, was studied with respect to further motions at seasonal time scales, revealing a wave with a semi-Chandler period found as a significant peak in the amplitude and power spectra as computed by Fast Fourier Transform and spectral analysis (Höpfner 1982, 1983, 1985). Using harmonic analysis, the residual latitudes at running intervals of 6 and 3 years were then used to calculate amplitude and phase values of the half-Chandler wobble. Here, referring to an observed mean Chandler period of 1.189 years (Höpfner 1980, 1983), the half-Chandler period was adopted to be 0.5945 years. For the phase variations, a linear trend was evident. Taking into consideration that this trend was caused by the small difference between the adopted and actual values of the period, an improved half-Chandler period of $(0.5839 \pm 0.0005)$ years or $(213.27 \pm 0.18)$ days was derived from the phase values affected with the trend (Höpfner 1982, 1983; Höpfner and Jochmann 1984).

Based on the combined time series EOP(IERS) C04 at daily intervals from 1976.5 to 1988.0, the parameters of the semi-annual and semi-Chandler wobbles together with those of the annual and Chandler wobbles for the trigonometic, exponential and elliptic representations were computed by Höpfner (1995, 1996a), using a semi-Chandler period of 206.19 days or 0.5645 years. The parameters of the ellipses obtained for the semi-Chandler and semi-annual wobbles are presented in Table 1. For illustrating the four wobbles, see Höpfner (1995, 1996a). Also the atmospheric excitation portions at annual and semi-annual frequencies were derived using the time series of the Atmospheric Angular Momentum $(\mathrm{AAM})$ functions, in particular $\chi(\mathrm{P}+\mathrm{IB})=\chi_{1}(\mathrm{P}+\mathrm{IB})+\mathrm{i} \chi_{2}(\mathrm{P}+\mathrm{IB})$. That is, the pressure terms which include an oceanic contribution using the Inverted-Barometer (IB) response, as computed by the U. S. National Meteorological Center (NMC).

Kosek and Kołaczek (1997) studied the semi-annual and semi-Chandler wobbles using the pole coordinate data computed by IERS for the period 1962.0 to 1996.0 and those computed by Vondrák et al. (1995) for the period 1900.0 to 1988.0. The pole paths of both separated oscillations were displayed for three intervals: 1983-1986, 1986-1989 and 1989-1992. Here the semi-Chandler period is given as 218 days.

For the semi-annual and semi-Chandler components of polar motion, it should be remarked: The semi-annual wobble has an elliptic prograde motion, where the direction of the semi-major axis is rather stable. On the contrary, the semiChandler wobble is much smaller, and the direction of the semi-major axis is extremely variable. Unfortunately, since there are no results over common intervals derived from the IERS data by Höpfner (1995) and Kosek and Kołaczek (1997), a direct comparison of results cannot be made. 
Based on the precise space-geodetic measurements, we have studied the major components of polar motion including the low-frequency component, the Chandler and annual terms (Höpfner, 2002). This work should be continued with a focus on the observed higher-frequency polar motions that are substantially smaller than the Chandler and annual wobbles.

\section{Data sets used in this study}

The combined Earth orientation series, SPACE99, as computed by the Jet Propulsion Laboratory (JPL) with one-day sampling (Gross, 2000) is used as input data, after removing the low-frequency, the Chandler and annual terms; see Höpfner (2002). These data of polar motion are referred to as the residual motions. Concerning their $x_{1}-$ and $x_{2}-$ components, note that the $x_{1}$-axis is in the direction of the IERS Reference Meridian (IRM) near the Greenwich meridian, and the $x_{2}$-axis is in the direction $90^{\circ} \mathrm{E}$ longitude. That is, we use the mathematical coordinate system with $x_{1}=\mathrm{x}$ and $x_{2}=-\mathrm{y}$ where $\mathrm{x}, \mathrm{y}$ are the coordinates of the Celestial Ephemeris Pole (CEP) relative to the IERS Reference Pole (IRP).

In order to give a visual impression of the total motions, sums of the low-frequency, Chandler and annual terms and residual motions, henceforth referred to as residual motions (1), these series are shown in Fig. 1, with those of the $x_{1}$-component in the upper part and of the $x_{2}$-component in the lower part. Note that the total polar motions as computed by JPL are given from MJD 43049.0 (1976 9 28.0) to 51565.0 (2000 1 22.0) whereas the resulting sums and, therefore, the residual motions (1) are obtained from MJD 44004.0 (1979 5 11.0) to 50610.0 (1997 6 11.0).

\section{Data processing and results}

In studying polar motions that are substantially smaller than the Chandler and annual wobbles, the data processing is given as a flow chart in Fig. 2 and includes the following four steps:

(1) To obtain the periodic signals contained within the residual motions (1), the amplitude spectrum (1) of them is computed by Fast Fourier Transform (FFT) and is presented in Fig. 3 (top-left). The semi-annual, semi-Chandler and 4-month signals can be seen as distinct peaks with periods of 183, 225 and 120 days respectively. Then, separating the semi-Chandler and semi-annual terms by recursive band-pass filtering and the 4-month term by band-pass filtering of the residual motions (1). To isolate these and the other terms considered later, we designed the band-pass filters according to Höpfner (1996b). Figure 4 presents the resulting semi-Chandler, semi-annual and 4-month oscillations, with those for the $x_{1}$-component in the upper part and those for the $x_{2}$-component in the lower part.

(2) Calculating the residual motions (2) that remain after removing the terms of (1) and amplitude spectrum (2) of them that reveals the other periodic signals shown in Fig. 3 (top-right). In particular, see the 2-month and 1.5-month signals with periods of 60 and 45 days and the quasi-biennial signal with a period of 650 days. The 2-month and 1.5month terms are then separated by recursive band-pass filtering, and the quasi-biennial term by band-pass filtering of the residual motions (2). These terms are presented in Fig. 5, separately for the $x_{1}-$ and $x_{2}-$ components in the upper and lower parts respectively.

(3) Calculating the residual motions (3) that remain after the removal of the terms of (2) and amplitude spectrum (3) of them that reveals the other periodic signals as shown in Fig. 3 (bottom-left). In particular, the 300-day and 90-day signals can be seen which are then separated by band-pass filtering from these series. Figure 6 displays the residual motions (3) and the oscillations filtered out with periods of 300 and 90 days, again in the upper part for the $x_{1}-$ component and in the lower part for the $x_{2}$-component.

(4) Computing the residual motions (4) as the remaining motions after the removal of the terms of (3) and amplitude spectrum (4) of them that reveals the other periodic signals shown in Fig. 3 (bottom-right). The residual motions (4) are also presented in Fig. 6 (bottom panels).

As noted, in Fig. 1, we can see the residual motions (1) that are computed as difference series of the total motions and the sums of the low-frequency, Chandler and annual terms. The amplitude spectra of all residual motions (1) to (4) are computed by FFT and displayed in Fig. 3. Figures 4 to 6 show to what extent the periodic terms filtered out are irregularly occurring in the $x_{1}-$ and $x_{2}$-components. Using the space-time view of Höpfner (1994), Fig. 7 presents the quasi-biennial, 300-day, semi-Chandler and semi-annual motions in terms of elliptic spiral curves, while the same is shown in Fig. 8 for the 4-month, 90-day, 2-month and 1.5-month motions. Some characteristics of these oscillations, including the time interval of the resulting series, period length, type, semi-major axis and its direction, are listed in Table 2. 
Publication: Scientific Technical Report

No.: 02/13
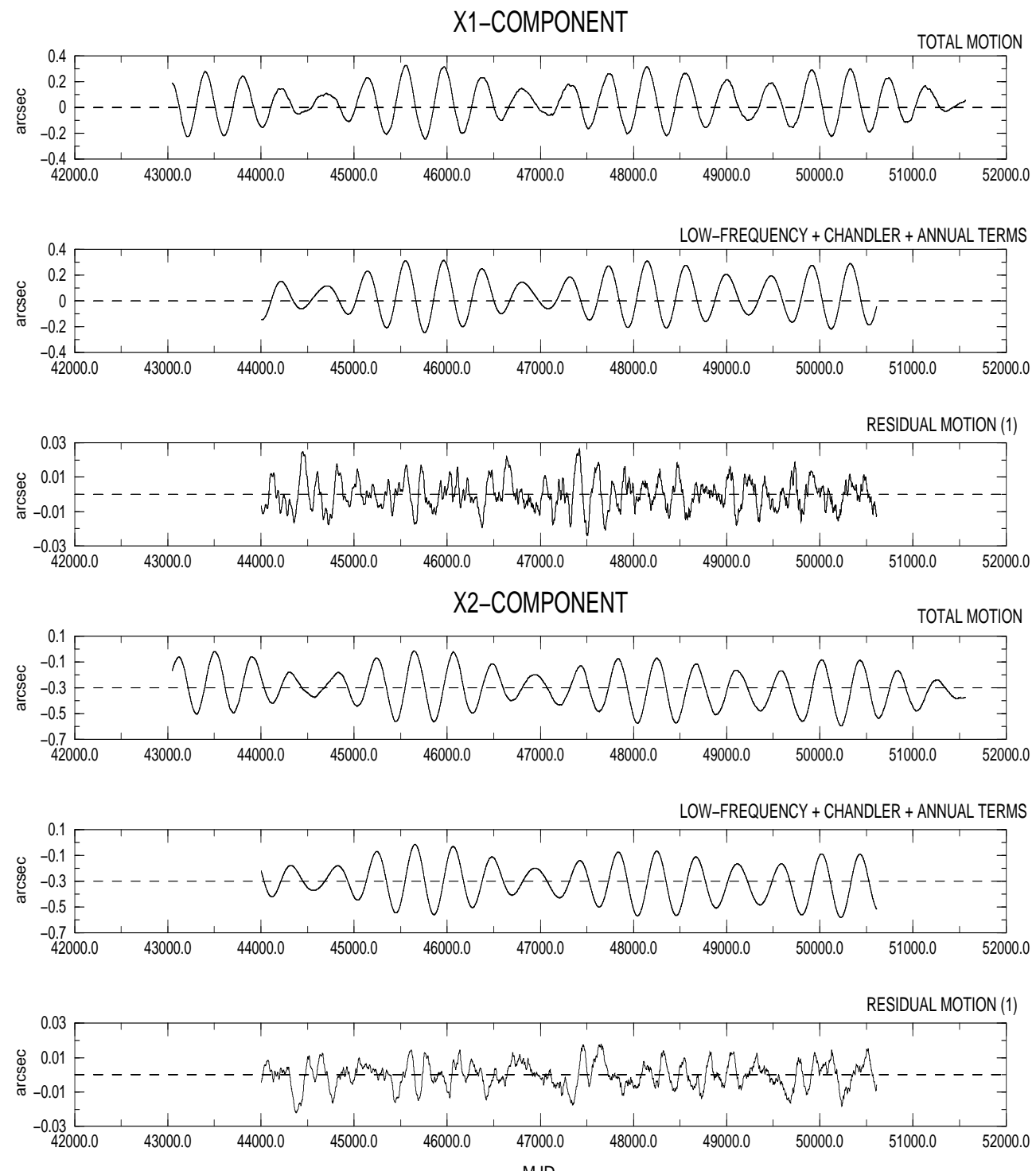

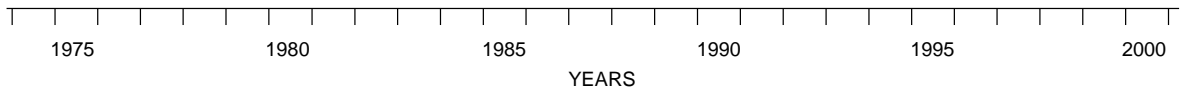

Figure 1. Polar motion in the $x_{1}$-component (upper part) and the $x_{2}-$ component (lower part). In each part, the motions shown are: Total motion as computed by JPL (top), the sum of the low-frequency, Chandler and annual terms (centre), and the residual motion (1)(bottom).

Table 2. Characteristics of the periodic components of polar motion that are substantially smaller than the Chandler and annual wobbles

\begin{tabular}{|c|c|c|c|c|c|c|}
\hline Component & $\begin{array}{l}\text { Time interval } \\
\text { (MJD) }\end{array}$ & $\begin{array}{l}\text { Time interval } \\
\text { (calendar days) }\end{array}$ & $\begin{array}{l}\text { Period } \\
\text { (days) }\end{array}$ & Type & $\begin{array}{l}\text { Semi-major } \\
\text { axis }(\operatorname{arcsec})\end{array}$ & $\begin{array}{l}\text { Direction of the } \\
\text { semi-major axis }\end{array}$ \\
\hline Quasi-biennial & $45861.0 \ldots 48933.0$ & $1983 / 12 / 13 \ldots$ 1992/11/07 & $600 \ldots 750$ & variably elliptic & $0.003 \ldots 0.008$ & $70^{0} \ldots 110^{0}$ \\
\hline 300-day & $47056.0 \ldots 47558.0$ & 1987/09/18 ... 1989/02/01 & $290 \ldots 300$ & clearly elliptic & about 0.004 & about $170^{0}$ \\
\hline Semi-Chandler & $44466.0 \ldots 50148.0$ & 1980/08/15 ... 1996/03/06 & $200 \ldots 250$ & elliptic to circular & $0.004 \ldots 0.008$ & very variable \\
\hline Semi-annual & $44397.0 \ldots 50217.0$ & 1980/06/07 ... 1996/05/14 & $175 \ldots 190$ & elliptic & $0.004 \ldots 0.013$ & $10^{0} \ldots 35^{0}$ \\
\hline 4-month & $44728.0 \ldots 49886.0$ & $1981 / 05 / 04 \ldots 1995 / 06 / 18$ & $110 \ldots 135$ & elliptic to circular & $0.001 \ldots 0.006$ & about $150^{0}$ \\
\hline 90-day & $46641.0 \ldots 47937.0$ & 1986/07/30 ... 1990/03/23 & $80 \ldots 95$ & elliptic & $0.001 \ldots 0.003$ & very variable \\
\hline 2-month & $44859.0 \ldots 49755.0$ & $1981 / 09 / 12 \ldots 1995 / 02 / 07$ & $55 \ldots 70$ & clearly elliptic & $0.001 \ldots 0.005$ & very variable \\
\hline 1.5-month & $44827.0 \ldots 49787.0$ & 1981/08/11 ... 1995/03/11 & $40 \ldots 55$ & clearly elliptic & $0.001 \ldots 0.004$ & about $170^{0}$ \\
\hline
\end{tabular}


Publication: Scientific Technical Report

No.: 02/13

Author: J. Höpfner

(1) Residual motions (1)

Fast Fourier Transform: Amplitude spectrum (1)

Separating the semi-Chander, semi-annual and 4-month terms

(2) Calculating the residual motions (2)

Fast Fourier Transform: Amplitude spectrum (2)

Separating the 2-month, 1.5-month and quasi-biennial terms

(3) Calculating the residual motions (3)

Fast Fourier Transform: Amplitude spectrum (3)

Separating the 300-day and 90-day terms

(4) Calculating the residual motions (4)

Fast Fourier Transform: Amplitude spectrum (4)

Figure 2. Flow chart about the four steps of data processing.
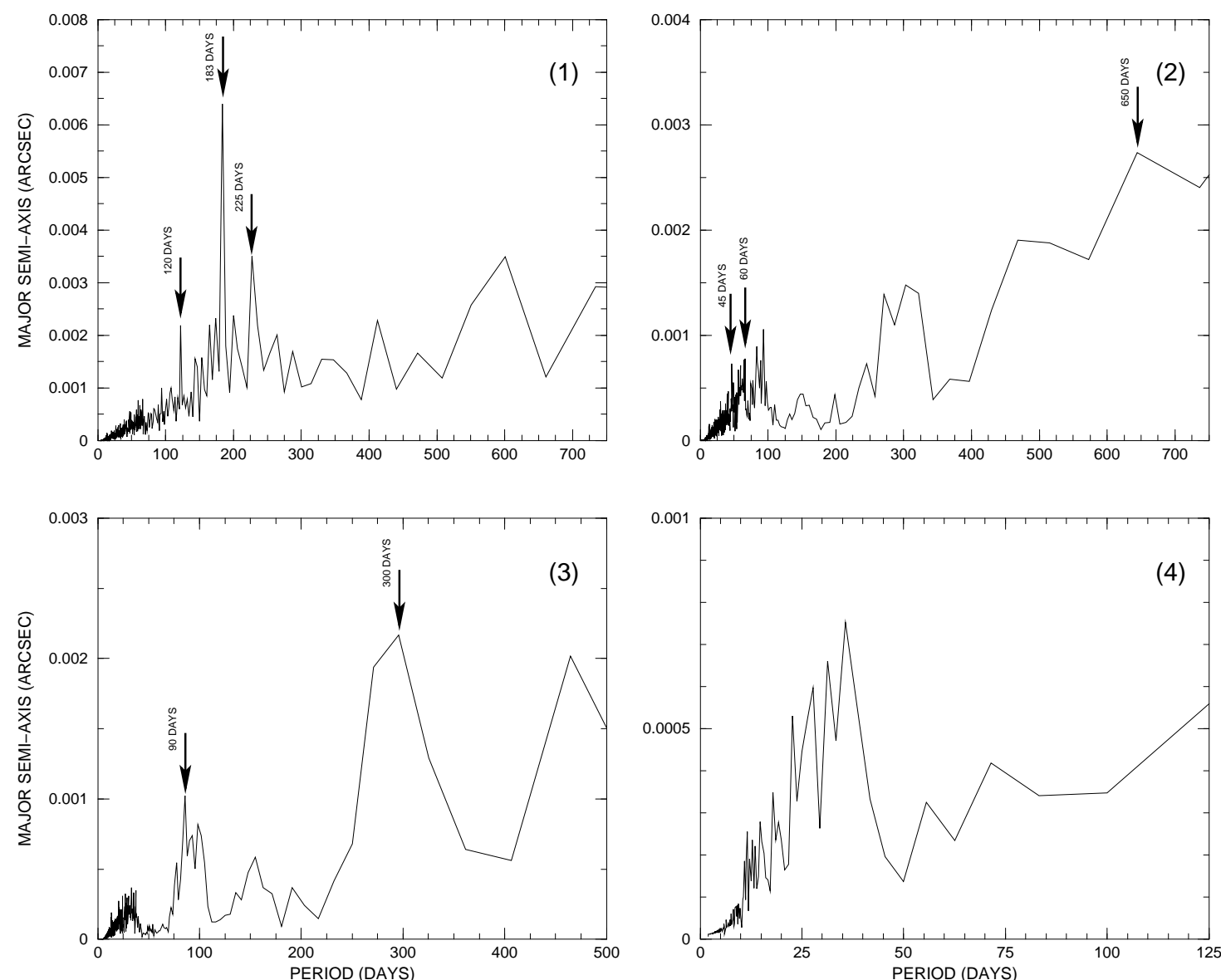

Figure 3. Amplitude spectra of the residual motions (1), (2), (3), and (4). The arrows indicate the periodic terms that have been separated out by band-pass filtering of the residual motions (1), (2), and (3), respectively. 
Publication: Scientific Technical Report

No.: 02/13

Author: J. Höpfner
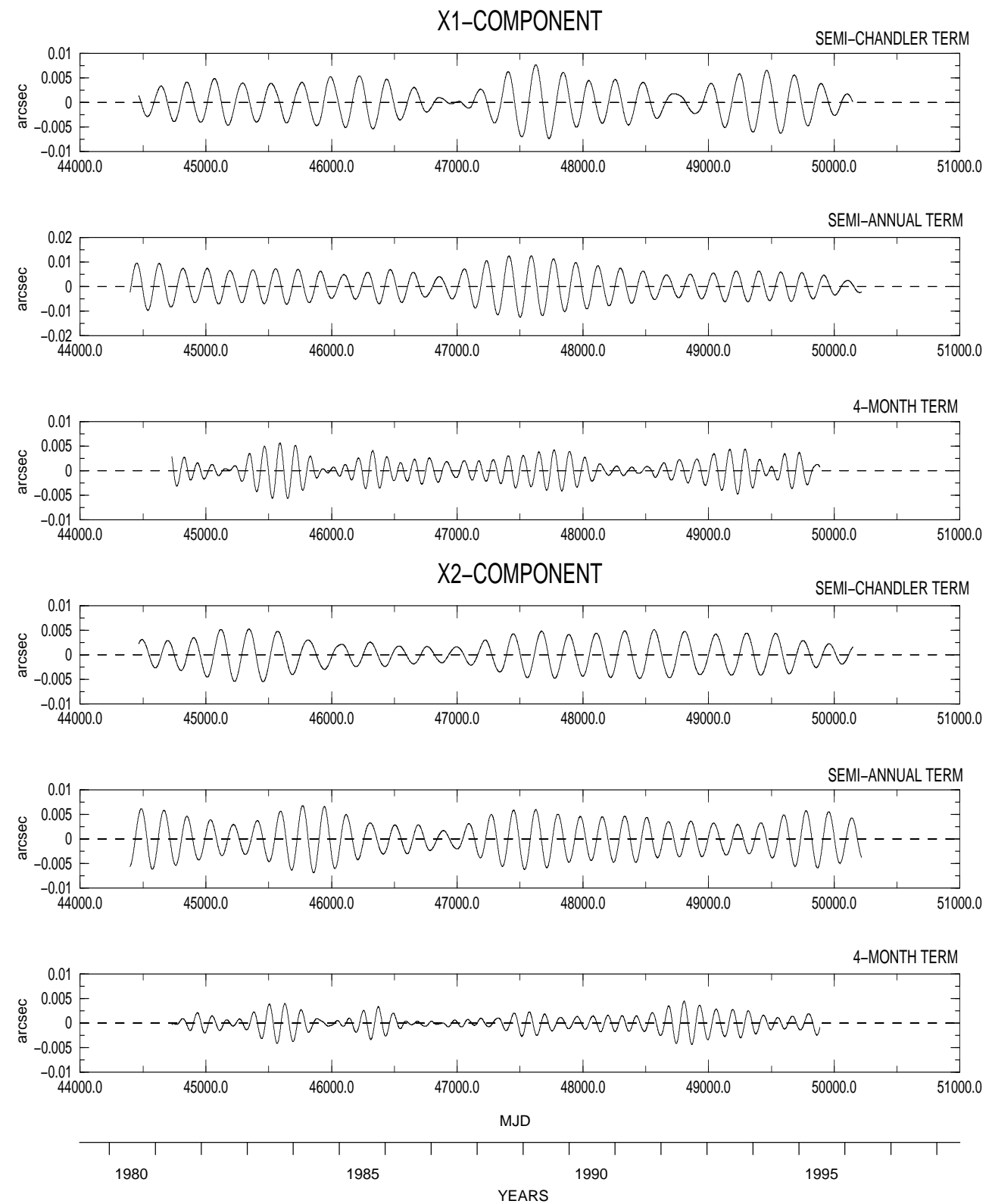

Figure 4. Polar motion in the $x_{1}$-component (upper part) and the $x_{2}$-component (lower part). In each part, the motions shown are: The semiChandler term (top), the semi-annual term (centre), and the 4-month term (bottom). 
Publication: Scientific Technical Report

No.: 02/13

Author: J. Höpfner
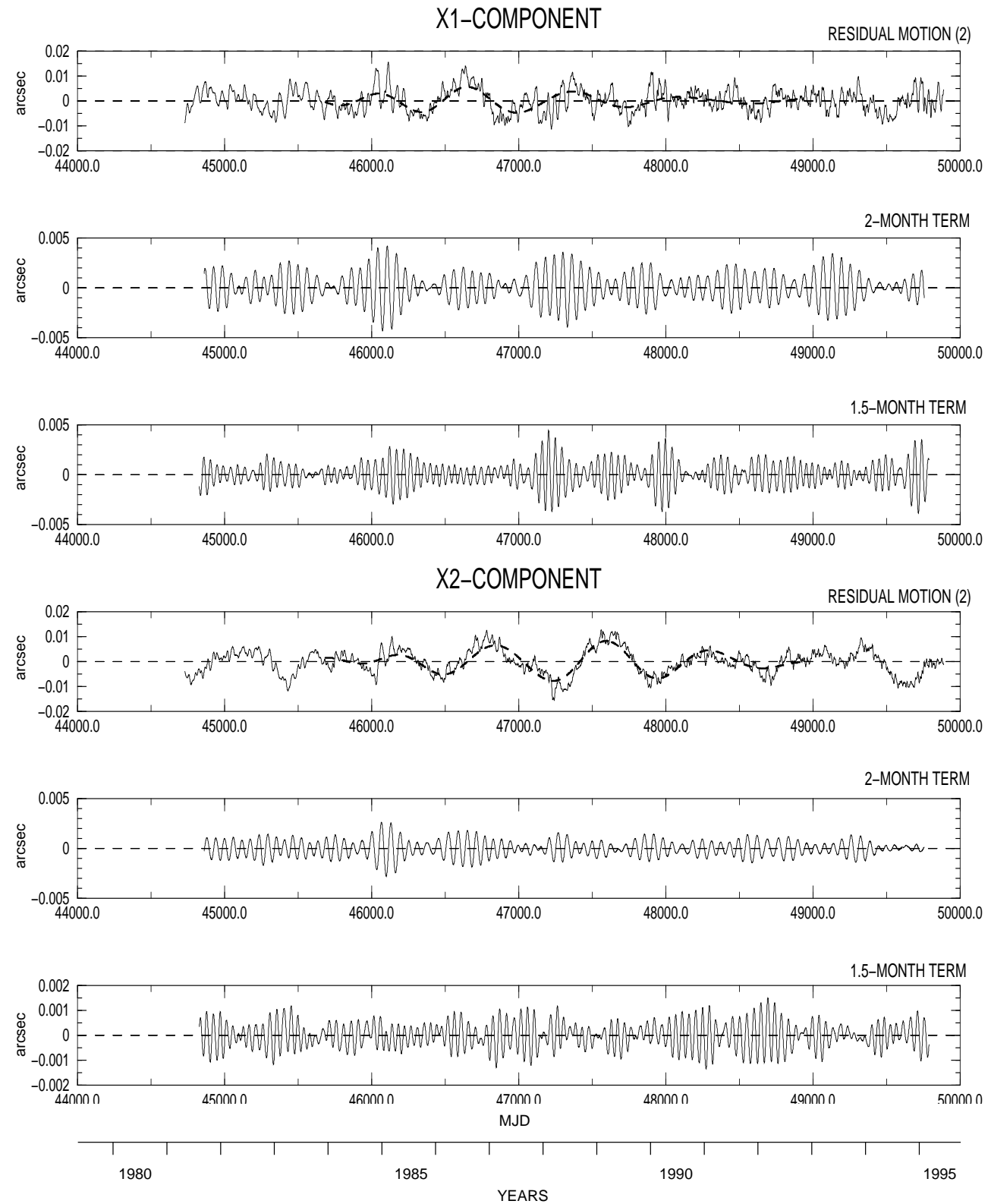

Figure 5. Polar motion in the $x_{1}$-component (upper part) and in the $x_{2}$-component (lower part). In each part, the motions shown are: Residual motion (2) (top), the 2-month term (centre), and the 1.5-month term (bottom). In the top panels, the quasi-biennial term is plotted by a dashed line. 
Publication: Scientific Technical Report

No.: 02/13

Author: J. Höpfner
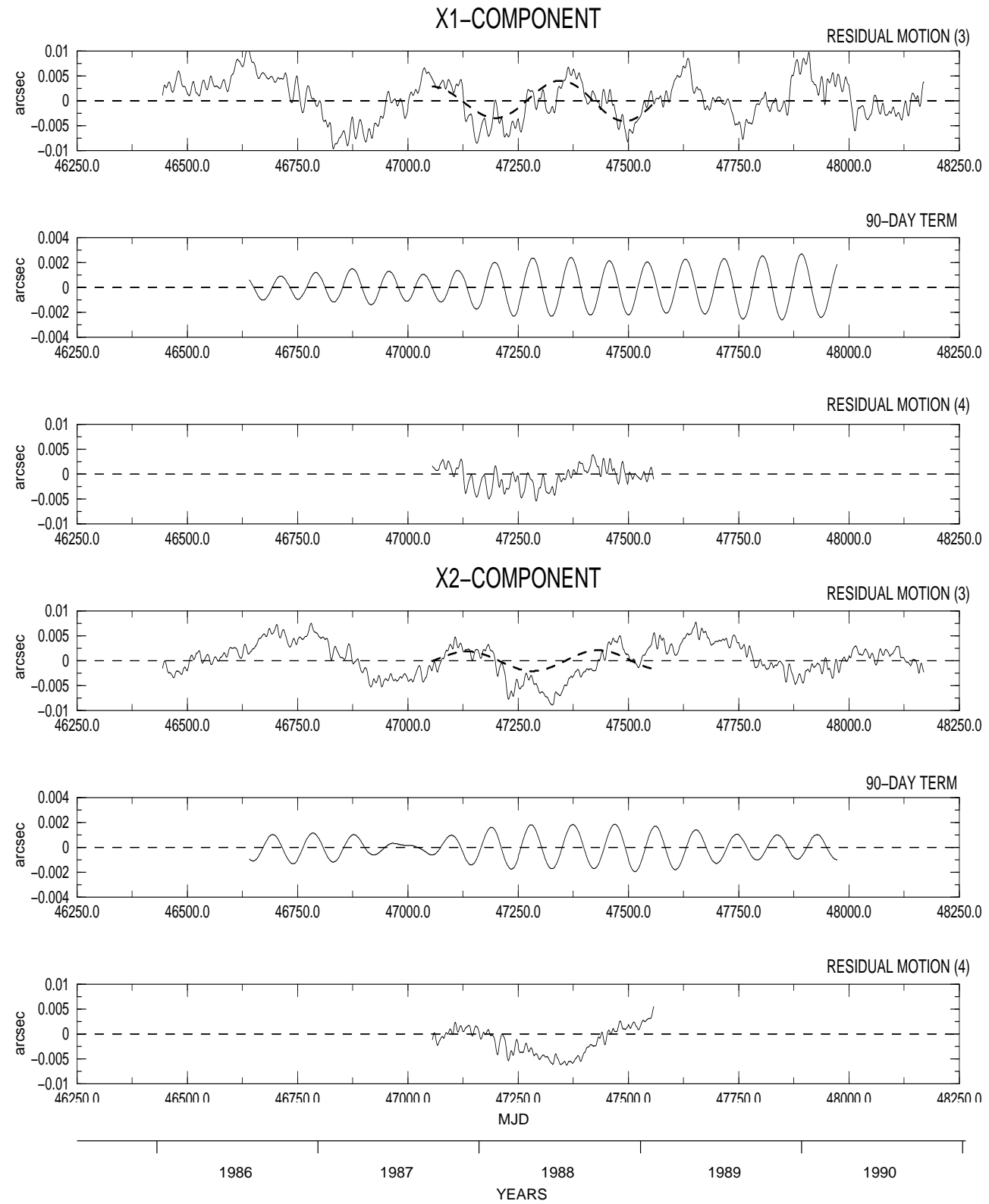

Figure 6. Polar motion in the $x_{1}$-component (upper part) and in the $x_{2}$-component (lower part). In each part, the motions shown are: Residual motion (3) (top), the 90-day term (centre), and residual motion (4) (bottom). In the top panels, the 300-day term is plotted by a dashed line. 
Publication: Scientific Technical Report

No.: 02/13

Author: J. Höpfner

QUASI-BIENNIAL WOBBLE

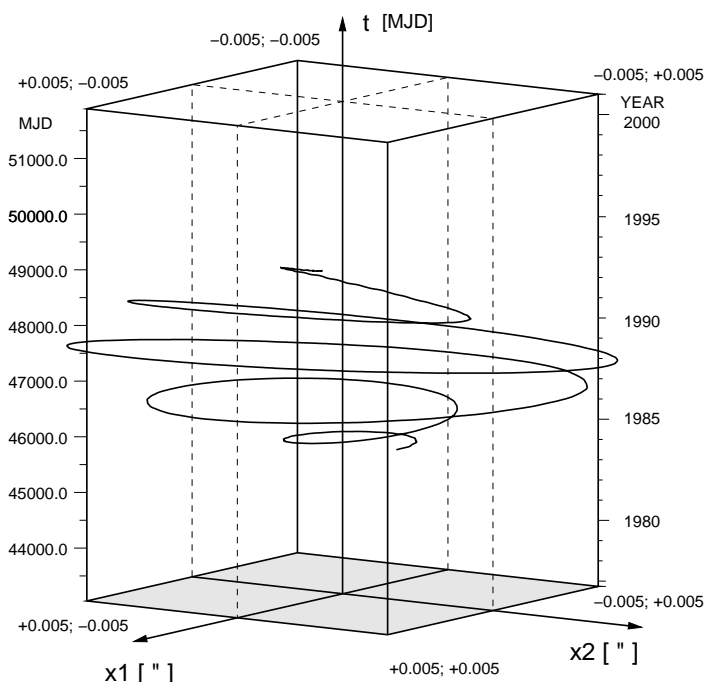

SEMI-CHANDLER WOBBLE

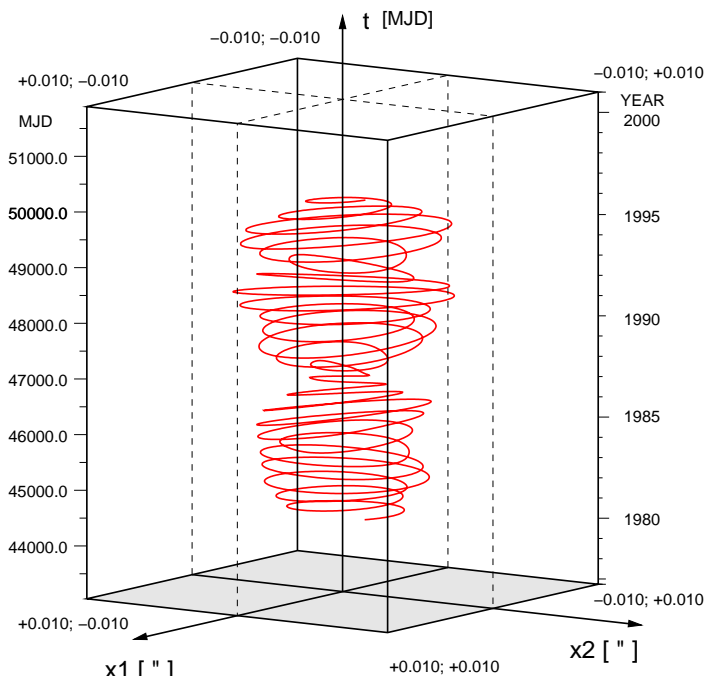

300-DAY WOBBLE

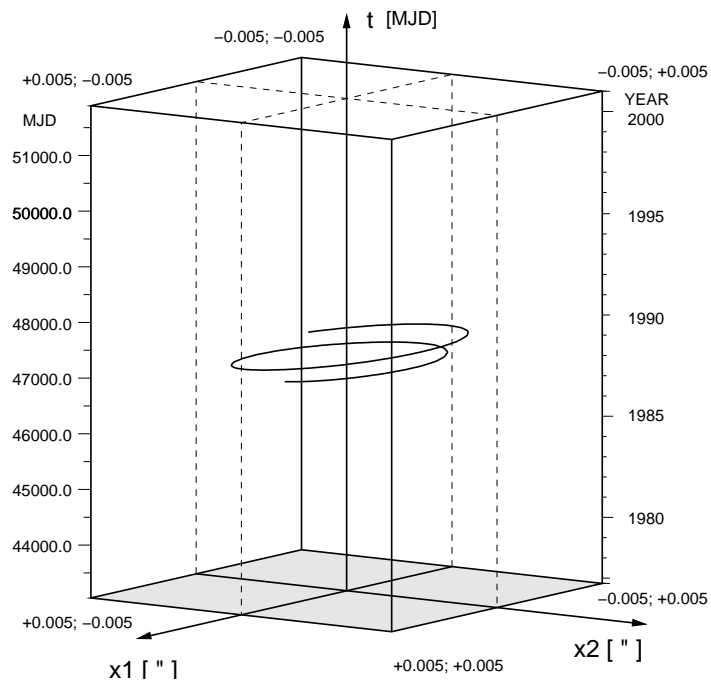

SEMI-ANNUAL WOBBLE

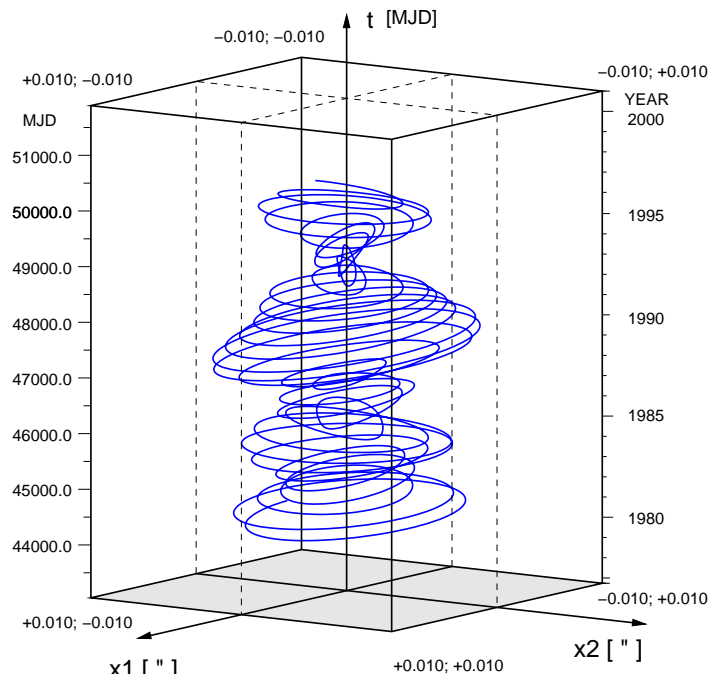

$\mathrm{x} 1[\mathrm{~s}]$

$+0.010 ;+0.010$

Figure 7. Quasi-biennial, 300-day, semi-Chandler, and semi-annual wobbles filtered out from the series EOP (JPL) SPACE99 using the space-time view of Höpfner (1994). The $x_{1}$-axis points towards the Greenwich meridian, and the $x_{2}-$ axis towards $90^{\circ} \mathrm{E}$ longitude. 
Publication: Scientific Technical Report

No.: 02/13

Author: J. Höpfner

\section{4-MONTH WOBBLE}

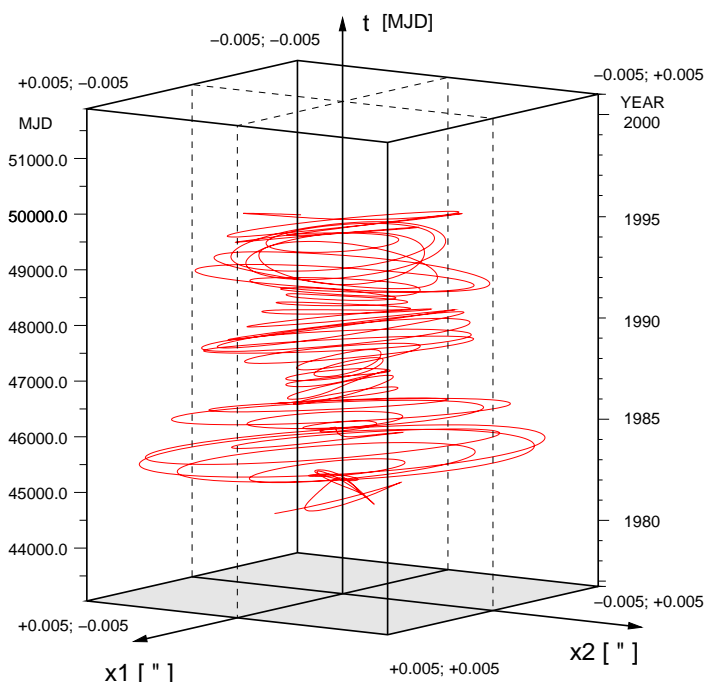

2-MONTH WOBBLE

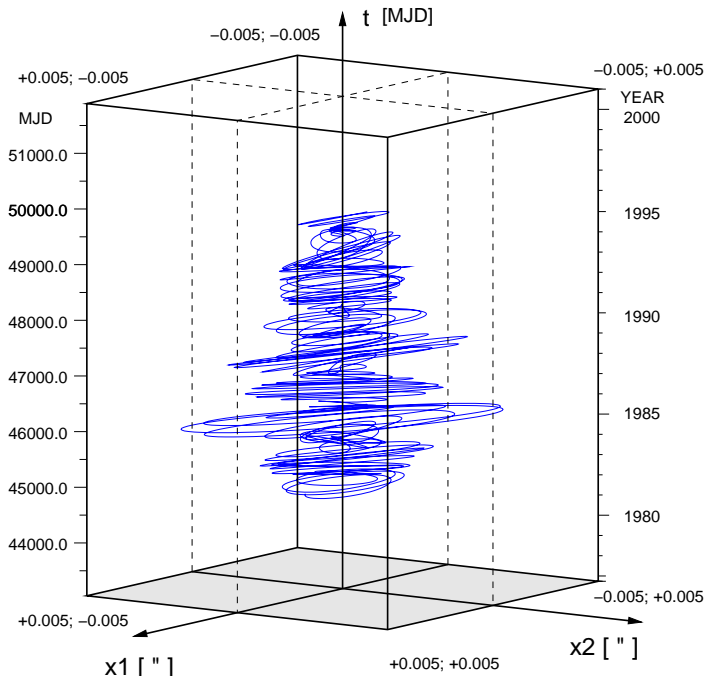

90-DAY WOBBLE

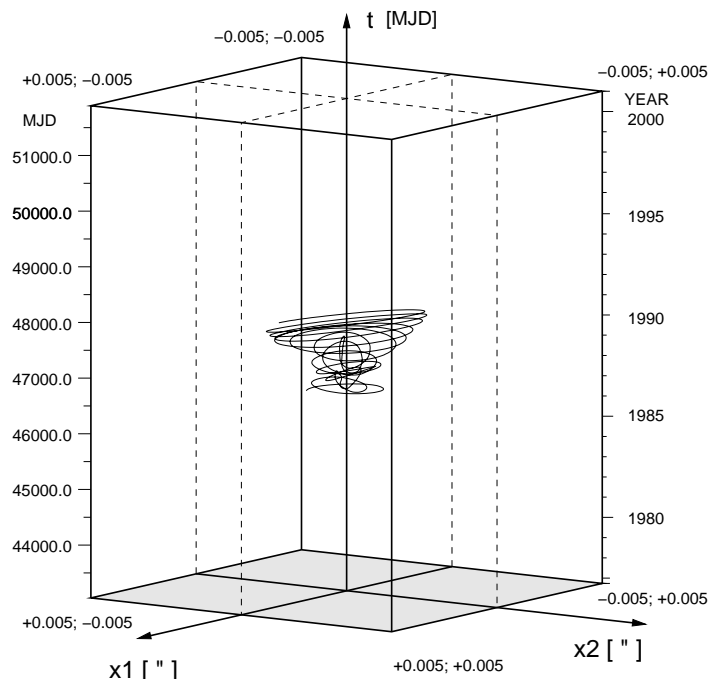

1.5-MONTH WOBBLE

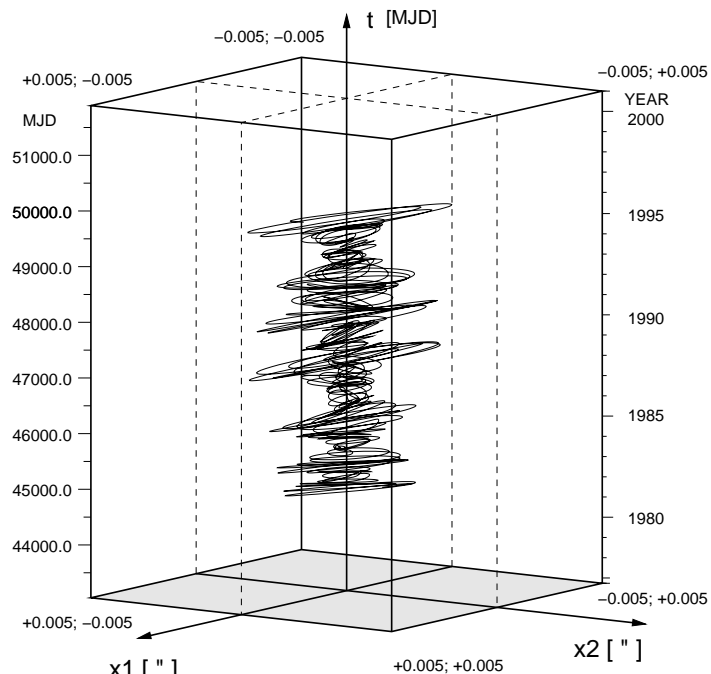

x1 [" ]

$+0.005 ;+0.005$

Figure 8. 4-month, 90-day, 2-month, and 1.5-month wobbles filtered out from the series EOP (JPL) SPACE99 using the space-time view of Höpfner (1994). The $x_{1}$-axis points towards the Greenwich meridian, and the $x_{2}$-axis towards $90^{0}$ E longitude. 


\section{Discussion of the results}

Using the amplitude spectra of the residual motions (1) to (3) as shown in Fig. 1, we assess them with respect to periodic signals for their separating. The arrows mark in each spectrum those signals that are revealed and accordingly have been separated. As the amplitude spectrum of the residual motions (4) shows, there is still a very small 1-month signal.

From Table 2, we can see that all of the PM signals filtered out from the remaining motions after the low-frequency, Chandler and annual terms have been removed (especially from SPACE99 computed by JPL; Höpfner, 2001), are oscillations with variable amplitudes reaching maxima of 3 to 13 mas over their analysis intervals. From Figs. 6 and 7, we note that the motions occur progradely (i.e. counter-clockwise), similar to the Chandler and annual wobbles. Note also that the persistence of the oscillations becomes less with higher frequencies. The filtered terms are now to be discussed in detail.

Over a relatively short analysis interval of ca. 9 years, there are 4.5 revolutions of the quasi-biennial wobble showing considerable variability in the amplitude (semi-major axis), in particular increasing from 3 to 8 mas at 1989 after which it decreases again to less than 3 mas, while not having the stable period as given in Table 2 . The orientation of the elliptic motion, referred to the semi-major axis, lies between $70^{\circ}$ and $110^{\circ}$ with the type of quasi-biennial wobble being very variably elliptic.

The 300-day wobble found over only about 500 days has a clearly elliptic motion with a semi-major axis of ca. 4 mas in the direction of ca. $170^{\circ}$ and a semi-minor axis of about 2 mas.

In the analysis interval from 1980 to 1996, the semi-Chandler wobble shows a motion varying from elliptic to circular with an amplitude of the order of 5 mas. Especially around 1989, there is a maximum amplitude of 8 mas whose direction is $15^{\circ}$. Two other maxima are in 1985 and 1994, with an amplitude of 6 mas at $160^{\circ}$ and 7 mas at $160^{\circ}$. In $1986 / 1987$ and 1996, distinct irregularities exist where the amplitude is much smaller, in particular about 2 mas.

Similarly, estimates for the semi-annual wobble were obtained for the period 1980-1996. From the elliptic spiral curve of this wobble, shown in Fig. 6 (bottom-right), we notice considerable variability in the amplitude (semi-major axis) with minima in 1982, 1985-1987 and 1992/1993 and maxima in 1980, 1984, 1988/1989 (where the amplitude reaches a magnitude of ca. 13 mas at a direction of ca. $10^{\circ}$ ) and 1995 . Here, the orientation of the elliptic motion is relatively stationary with stable ellipticity.

During 1981-1995, the 4-month wobble varies considerably both in amplitude and ellipticity. Especially in 1983/1984, the amplitude reaches a maximum of about 6 mas at a direction of about $160^{\circ}$ and in 1982 and again 1985 , a minimum of 1 mas. We find that the main orientation of the elliptic motion referred to the semi-major axis lies at about $150^{0}$.

Over 3.5 years between 1986 to 1990, we see the elliptic spiral curve of the 90-day wobble in Fig. 7 (top-right) showing a similar variability in both amplitude and ellipticity as the 4-month wobble. However, the amplitude of the 90day wobble is smaller, in particular varying between 1 and 3 mas, and its direction very variable. We can see amplitude maxima in 1988 and 1989 , each with 3 mas but with directions of $220^{\circ}$ and $160^{\circ}$.

The 2-month wobble found over a 13.5-year period from 1981 to 1995 shows three obvious maxima in 1985,1988 and 1993 of 5, 4 and 3 mas, with the direction of the semi-major axis lying at $150^{\circ}, 160^{\circ}$ and $10^{\circ}$ and five minima of nearly zero mas in 1983/1984, 1985/1986, 1987, 1990 and 1994. Generally, for the orientation of the elliptic motion, there is again much variability.

Like the 2-month wobble, the 1.5-month motion is filtered out over about 13.5 years from 1981 to 1995 . Its variability is characterised by alternating smaller and larger amplitudes ranging between 1 and 4 mas. In the case of the larger amplitudes of 3 to 4 mas (in 1985, 1988, 1990 and 1995), the orientation of the elliptic motion referred to the semi-major axis lies at about $170^{\circ}$.

Especially for information on the period variability of the elliptic motions given in Table 2, we found that the period of the semi-annual wobble varies by 15 days. The other higher-frequency wobbles also show a similar variation in their periods. Compared to it, the period of the semi-Chandler wobble has a 50-day variation interval, ranging from 200 to 250 days. Concerning the quasi-biennial and 300-day motions computed for relatively short time intervals, it should be noted that this is due to the band-pass filter method sequentially applied for this analysis according to the magnitude of a periodic signal revealed by FFT. Therefore, the estimates of these periodic components are secondary results of our paper.

Finally, some comments should be given about the geophysical explanation of the oscillations found by our analysis. Today, it is clear that all three excitation sources and in particular atmosphere, oceans, and hydrology provide contributions to the excitation of polar motion; see e.g. Wilson (2000). Note that there are a large number of papers on correlations between variations of polar motion and atmospheric excitation functions, for example Chao (1993), Kuehne et al. (1993), Nastula et al. (1997), Kołaczek et al. (2000), and further references therein. Especially at annual and semi-annual frequencies, the problem of the excitation was considered in the way that the total excitation is composed of atmospheric and non-atmospheric excitation portions (Höpfner 1996a). Using three ocean circulation models, oceanic contributions to seasonal polar motions are studied in Wünsch (2000). Schmitz-Hübsch and Schuh (1999) found that the semi-annual 
prograde variations are delayed by strong El Niño events. For regional atmospheric angular momentum contributions to polar motion excitation, see e.g. Salstein and Rosen (1989), Nastula and Salstein (1999). Based on a constant-density, nearly global numerical ocean model, the results of the oceanic excitation of signals in the Earth's rotation are presented in Ponte (1997) and Ponte et al. (1998), indicating that the oceanic circulation and mass-field variability play important roles in the excitation of seasonal to fortnightly polar motion. Studies dealt with the hydrological contributions to polar motion excitation are made, for example, by Chao et al. (1987), Chao and O'Connor (1988), Wilson and Kuehne (1990), and Wilson (1993). Using the wavelet analysis, seasonal and short-period variations in the Earth's rotation measured by VLBI from 1981 to 1999 are investigated by Schmidt and Schuh (2000), and Schuh and Schmitz-Hübsch (2000). Comparing the periods of polar motion and atmospheric angular momentum time series, it was found that irregular shortperiod PM variations, in particular between two and five months, are partially caused by atmospheric excitations with a normed coherency between 0.3 and 0.6 .

Recent studies of PM including its excitation are published in the proceedings of the IAU Colloquium 178 "Polar motion: Historic and Scientific Problems" held at Cagliari, Italy in September 1999 to celebrate the 100th anniversary of the International Latitude Service (ILS), see Dick et al. (2000) and in particular the papers given by Wilson (2000), Salstein (2000), Nastula et al. (2000), and Kołaczek et al. (2000).

\section{Concluding remarks}

Estimates obtained from different methods should be similar to each other in order to resolve significant signals. For a comparison of our estimates relative to the JPL system with others, see Kosek et al. (1995), Kosek and Kołaczek (1997), and Kołaczek et al. (2000) for temporal variability, and Höpfner (1995, 1996a) for means over the study period between 1976 and 1987. Comparing the estimates, we find that they are similar in the general form but with some disagreements in the details. These differences will be elucidated in the future. In this work, since the comparison estimates are relative to the IERS system, small discrepancies are explainable by systematic errors, i.e. due to different approaches used by JPL and IERS to correct the bias and rate of the individual series before they are combined.

Acknowledgements. Some information of this paper was presented at the XXVI General Assembly of the European Geophysical Society in Nice, France, 26-30 March 2001. Thanks to Kevin Fleming for his linguistic advice. Also I would like to thank Harald Schuh for his helpful comments.

\section{References}

Albrecht, Th. and Wanach, B., Resultate des Internationalen Breitendienstes, Bd. III. Z. B. Internat. Erdmessung N. F. Veröff. Nr. 18. Berlin, 1909. Chao, B. F., Excitation of Earth's polar motion by atmospheric angular momentum variations, 1980-1990, Geophys. Res. Lett., $20,253-256,1993$.

Chao, B. F., O'Connor, W.O., Global surface-water-induced seasonal variations in the Earth's rotation and gravitational field, Geophys. J., 94, 263-270, 1988.

Chao, B. F., O'Connor, W. P., Chang, A. T. C., Hall, D. K. and Foster, J. L., Snow-load effect on the Earth's rotation and gravitational field, $1979-1985$. J. Geophys. Res., 92, B9, 9415-9422, 1987.

Dick, S., McCarthy, D. and Luzum, B. (eds.), Polar motion: Historical and scientific problems, ASP Conf. Ser. Vol. 208.641 pp., 2000.

Emetz, A. I., Study of the spectrum of the latitude variations in the frequency range between 1.4 and 2.2 cpy and search of a nearly-daily polar motion (in Russian), Astrometrija i astrofizika, 38, 84-95, 1979.

Emetz, A. I., Spectrum of the latitude variations in the frequency range between 1.4 and 2.2 cpy and search for nearly diurnal wobble. In: Fedorov, E. P., Smith, M. L., Bender, P. L. (eds.): Nutation and the Earth's rotation, D. Reidel Publ., pp. 75-79, 1980.

Fedorov, E. P., Korsun, A. A., Major, S. P., Panchenko, N. I., Taradi, V. K., Yatskiv, Ya. S., Polar motion of the Earth from 1890.0 to 1969.0 (in Russian), Ukrainian Acad. Sc. Kiev, 1972.

Gross, R. S., Combinations of Earth Orientation Measurements: SPACE99, COMB99, and POLE99, JPL Publication 00-5, Pasadena, California, 2000.

Helmert, F. R.; Albrecht, Th., Der internationale Polhöhendienst, Astron. Nachr.,148, 3532, 49-56, 1899.

Höpfner, J., Analysis of long-term latitude determinations at Potsdam Observatory with respect to variations in their principal components,Gerlands Beitr. Geophysik,86, 6, 449-459, 1977.

Höpfner, J., Mean latitude, Chandler, annual and semiannual wobbles determined at Potsdam Observatory in their refined variability with time, Gerlands Beitr. Geophysik, 88, 3, 185-192, 1979.

Höpfner, J., Variation der Chandler-Periode aus den am Observatorium Potsdam beobachteten Breiten, Gerlands Beitr. Geophysik, 89, 3/4, 182-186, 1980.

Höpfner, J., On the wave with the semi-Chandler period obtained from the Potsdam latitude observations (in Russian). In: Proc. of the 1st Orlov Conf. 1980, Naukova Dumka, Kiev, pp. 149-153, 1982.

Höpfner, J., Umfassende Analyse der Breitenbestimmungen des geodätisch-astronomischen Observatoriums Potsdam, Diss. B. Techn. Univ. Dresden, 177 p., 1983.

Höpfner, J., Untersuchungen langjähriger Potsdamer Breitenbestimmungen auf mittelperiodische Schwankungen, Vermess.-Techn., 33, 4, 112-114, 1985 .

Höpfner, J., Zur Veranschaulichung von zweidimensionalen geophysikalischen Prozessen und ihren periodischen Anteilen, AVN, 101,2 , 45-55, 1994.

Höpfner, J., Periodische Anteile in der Erdrotation und dem atmosphärischen Drehimpuls und ihre Genauigkeiten, ZfV, 120, 1, 8-16, 1995.

Höpfner, J., Polar motion at seasonal frequencies, J. Geodynamics, 22, 1/2, 51-61, 1996a.

Höpfner, J., Seasonal oscillations in length-of-day, Astron. Nachr., 317, 4, 273-280, 1996 b.

Höpfner, J., The International Latitude Service - a historical review, from the beginning to its foundation in 1899 and the period until 1922 , Surveys in Geophysics, 21, 5/6, 521-566, 2000.

Höpfner, J., Chandler and annual wobbles based on space-geodetic measurements. This issue, 2002. 


\section{Publication: Scientific Technical Report}

No.: 02/13

Author: J. Höpfner

Höpfner, J., Jochmann, H., Cause and evidence of the half-Chandler wobble, Proc. IAG, Vol. 2, 100-107, 1984.

IERS, 1998 Annual report, Obs. de Paris, 1999.

Kołaczek, B., Kosek, W., Schuh, H., Short-period oscillations of Earth rotation. In: Dick, S., McCarthy, D. and Luzum, B. (eds.), Polar motion: Historical and scientific problems, ASP Conf. Ser. Vol. 208, 533-544, 2000.

Kosek, W., Nastula, J., Kołaczek, B., Variability of polar motion oscillations with periods from 20 to 150 days in 1979-1991, Bull. Geodesique, 69, 308-319, 1995.

Kosek, W., Kołaczek, B., Semi-Chandler and semi-annual oscillations of polar motion, Geophys. Res. Lett., 24, $2235-2238,1997$.

Kuehne,J., Johnson, S., Wilson, C. R., Atmospheric excitation of nonseasonal polar motion, J. Geophys. Res., 98, B11, 19973-19978, 1993.

Nastula, J., Kosek, W., Kołaczek, B., Analyses of zonal atmospheric excitation functions and their correlation with polar motion excitation functions, Annales Geophysicae, 15, 1439-1446, 1997.

Nastula, J., Ponte, R.M., Salstein, D.A., Regional signals in atmospheric and oceanic excitation of polar motion. In: Dick, S., McCarthy, D. and Luzum, B. (eds.), Polar motion: Historical and scientific problems, ASP Conf. Ser. Vol. 208, 463-472, 2000.

Nastula, J., Salstein, D., Regional atmospheric angular momentum contributions to polar motion excitation, J. Geophys. Res., 104, B4, 7347-7358, 1999.

Nicolini, T. and Fichera, E., Risultati del Servizio Internazionale delle Latitudini dal 1941.06 al 1948.98, Vol. 10. Napoli, 1970.

Ponte, R.M., Oceanic excitation of daily to seasonal signals in Earth rotation: results from a constant-density numerical model, Geophys. J. Int., 130, 469-474, 1997.

Ponte, R.M., Stammer, D., Marshall, J., Oceanic signals in observed motions of the Earth's pole of rotation, Nature, 391, 476-479, 1998.

Salstein, D., Atmospheric excitation of polar motion. In: Dick, S., McCarthy, D. and Luzum, B. (eds.), Polar motion: Historical and scientific problems, ASP Conf. Ser. Vol. 208, 437-446, 2000.

Salstein, D.A., Rosen, R.D., Regional contributions to the atmospheric excitation of rapid polar motions, J. Geophys. Res., 94, D7, 9971-9978, 1989.

Schmidt, M., Schuh, H., Abilities of wavelet analysis for investigating short-period variations of Earth rotation, IERS Technical Note, No 28, 73-80, 2000 .

Schmitz-Hübsch, H., Schuh, H., Seasonal and short-period fluctuations of Earth rotation investigated by wavelet analysis, Festschrift for Erik W. Grafarend, ed. by F. Krumm and V.S. Schwarze, Technical Reports, Dep. of Geodesy and Geoinformatics, Report Nr. 1999.6-2, 421-431, 1999.

Schuh, H., Schmitz-Hübsch, H., Short period variations in Earth rotation as been by VLBI, Surveys in Geophysics, 21, 5/6, 499-520, 2000.

Vondrák, J., Ron, C., Pešek, I., Čepek, A., New global solution of Earth orientation parameters from optical astrometry in 1900-1990, Astron. Astrophys., 297, 899-906, 1995.

Wanach, B., Resultate des Internationalen Breitendienstes, Bd. V. Z. B. Internat. Erdmessung N. F. Veröff. Nr. 30. Berlin, 1916.

Wanach, B. and Mahnkopf, H., Ergebnisse des Internationalen Breitendienstes von 1912.0 bis 1922.7, Potsdam, 1932.

Wilson, C.R., Contributions of water mass redistribution to polar motion excitation, Contr. Space Geodesy to Geodynamics, $24,77-82,1993$.

Wilson, C.R., Excitation of polar motion. In: Dick, S., McCarthy, D. and Luzum, B. (eds.), Polar motion: Historical and scientific problems, ASP Conf. Ser. Vol. 208, 411-419, 2000. 2000.

Wilson, C.R., Kuehne, J., Air and water contributions to polar motion excitation. In: Boucher, C., Wilkins, G.A. (eds.), Earth rotation and coordinate reference frames, Springer, Berlin, 74-81, 1990.

Wünsch, J., Oceanic influence on the annual polar motion, J. Geodynamics, 30, 389-399, 2000.

Yumi, S. and Yokoyama, K., Results of the ILS in a homogeneous system 1899.9-1979.0, Publ. Central Bureau of the International Earth Rotation Service. Internat. Latitude Observ., Mizusawa, 1980. 